\title{
Anti-PR3 and anti-MPO antibodies are not present in sera of patients with pulmonary tuberculosis
}

\author{
I. Lima $\cdot$ R. C. Oliveira $\cdot$ M. S. Cabral $\cdot$ A. Atta $\cdot$ \\ S. Marchi $\cdot$ E. Reis $\cdot$ M. G. Reis $\cdot$ L. Barbosa $\cdot$ \\ M. B. Santiago
}

Received: 6 October 2013 / Accepted: 28 March 2014 / Published online: 10 April 2014

(C) Springer-Verlag Berlin Heidelberg 2014

\begin{abstract}
Anti-neutrophil cytoplasmic antibodies (ANCA) are autoantibodies directed to intracellular components of neutrophils and are present in several vasculitic syndromes. Recently, these autoantibodies have been described in other autoimmune disorders as well as in infectious diseases such as tuberculosis (TB). As there are some clinical similarities between TB and granulomatosis with polyangiitis, we searched for ANCA in a group of patients with proven TB. Patients with TB confirmed by chest X-ray and sputum bacilloscopy either before or within 30 days after beginning treatment were included in this study. Anti-MPO and anti-PR3 antibodies were studied using well-standardized ELISA kits (INOVA Diagnostics, Inc.). ANCA were also investigated by indirect immunofluorescence (IIF). Fifty TB patients ( 26 females, mean age $47.34 \pm 17$ years) were enrolled in the present study. No patient tested positive for ANCA by IIF, or anti-MPO or anti-PR3 antibodies by ELISA. Although previous studies have shown the presence of ANCA in some infectious diseases, the findings of the present study demonstrated the absence of such antibodies in TB. The discrepancy in the prevalence of ANCA in TB among different studies may be attributed to methodological factors and/or the genetic background of the studied populations.
\end{abstract}

I. Lima $\cdot$ M. B. Santiago $(\varangle)$

Escola Bahiana de Medicina e Saúde Pública, Rua Frei Henrique, 08, Nazaré, Salvador, Bahia CEP 40.000-000, Brazil

e-mail: mitter@svn.com.br

R. C. Oliveira $\cdot$ M. S. Cabral · A. Atta

Faculdade de Farmácia, Universidade Federal da Bahia, Salvador, Brazil

S. Marchi · E. Reis · M. G. Reis · L. Barbosa

Fundação Osvaldo Cruz, Salvador, Bahia, Brazil
Keywords ANCA $\cdot$ Anti-PR3 $\cdot$ Anti-MPO $\cdot$ Tuberculosis

\section{Introduction}

Anti-neutrophil cytoplasmic antibodies (ANCA) are autoantibodies directed to intracellular components of neutrophils, usually described in small vessel systemic vasculitis such as granulomatosis with polyangiitis (GPA), microscopic Polyangiitis and Churg-Strauss syndrome. In GPA, their specificity is to proteinase 3 (PR3) whereas in the other two types of vasculitis, the most common antigenic target is myeloperoxidase (MPO) [1].

These autoantibodies have also been described in other autoimmune disorders [2-4] as well as in infectious diseases [5-10] particularly tuberculosis (TB) [11-13]. As GPA shares some clinical features with $\mathrm{TB}$, in areas with high prevalence of this infectious disease, the positivity of these serologic tests may lead to a misdiagnosis and consequently wrong treatment. The aim of the present study was to investigate the frequency of these antibodies in a group of patients with proven pulmonary TB from an endemic area in Brazil.

\section{Patients and methods}

Patients either untreated or within 30 days after beginning treatment for pulmonary TB confirmed by chest X-ray and sputum bacilloscopy were included in this study. All patients were older than 18 years, voluntarily agreed to participate in this study and signed a consent form. The Ethics Committee of our institution approved the project. The included patients were submitted to a complete clinical evaluation including muscle skeletal manifestations, time of the diagnosis and medicine in use. 
ANCA were determined by indirect immunofluorescence (IIF) using a commercially available kit (EUROIMMUN) following the protocol summarized below: The sera to be tested were diluted 1:10 in phosphate buffered saline (PBS) and incubated on the slides for $30 \mathrm{~min}$ in a moist chamber; after this period, the slides were washed with PBS. Again, the slides were incubated in the moist chamber for $30 \mathrm{~min}$, this time with an antihuman IgG conjugate (goat). After washing with PBS, the slides were examined with a fluorescence microscope. Serum samples were also tested for the presence of antibodies to PR3 and MPO utilizing well-standardized kits according to the manufacturer's (INOVA Diagnostics Inc.) recommendations. Values above $20 \mathrm{U}$ are considered positive.

Quantitative variables were presented as mean \pm SD or median and interquartile range, and qualitative variables were expressed as percentages. For statistical analysis, a package program (SSPS for Windows version 18.0) was used.

\section{Results}

Fifty pulmonary TB patients [twenty-six (52\%) female, mean age 47.34 ( \pm 17 years)] were enrolled in the present study. Forty-six patients were in the beginning of TB treatment with rifampicin, isoniazid and pyrazinamide and had a median treatment time of 8 days (interquartile range 2-19). The majority of the patients (31) had no joint symptoms, whereas the others described some unspecific muscle skeletal pain without evidence of arthritis. The clinical and epidemiological characteristics of the patients are presented in Table 1.

None of the tested samples was positive for ANCA by IIF or had antibodies to MPO or PR3 by ELISA. The statistical power of 50 patients to detect at least a $10 \%$ prevalence of antibodies to MPO or PR3 is $94 \%$.

\section{Discussion}

Historically antibodies to cytoplasmic components of neutrophils detected by immunofluorescence were initially described in patients supposedly infected by Ross River virus in 1982 [14]. These patients had several clinical features of autoimmune disorders such as fever, arthralgia and myalgia, and some of them had glomerulonephritis. Later on, such antibodies were associated with GPA [15], and presently, it is well known that patients with GPA have antibodies with specificity to PR3 that gives the cytoplasmatic pattern of ANCA (C-ANCA), whereas antibodies to MPO with the perinuclear pattern of ANCA (P-ANCA) are seen frequently, although not exclusively in patients with Chung Strauss syndrome and microscopic polyangiitis [1].

Curiously, in the last few years, studies have presented conflicting results regarding ANCA positivity in infectious
Table 1 Clinical and epidemiological features of the tuberculosis patients $(n=50)$

\begin{tabular}{lc}
\hline Characteristics & $N(\%)$ \\
\hline Female & $26(52)$ \\
Positive bacilloscopy & $50(100)$ \\
Abnormal chest X-ray & $49(98)^{\mathrm{a}}$ \\
Tabagism & $21(42)$ \\
Alcoholism & $13(26)$ \\
Illicit drug use & $1(2)$ \\
Tuberculosis symptoms & \\
Fever & $18(36)$ \\
Weight loss & $17(34)$ \\
Cough & $50(100)$ \\
Hemoptysis & $5(10)$ \\
Chest pain & $6(12)$ \\
Dyspnoea & $9(18)$ \\
Tuberculosis treatment & \\
R/I/P & $46(92)^{\mathrm{b}}$ \\
Co-Morbidities & \\
HIV & $3(6)$ \\
Diabetes & $8(16)$ \\
Hypertension & $10(20)$ \\
\hline
\end{tabular}

a One patient did not have a chest X-ray

b Three patients had not started treatment, and one patient discontinued it due to intolerance

${ }^{\text {c }} R$ rifampicin/I isoniazid $/ P$ pyrazinamide

diseases, particularly pulmonary TB. As this condition bears some clinical and histopathological features of GPA, such as cough, fever, hemoptysis, nodular and cavitation lesions on chest imaging and chronic inflammation and granuloma at lung biopsy, it is mandatory to clarify this point, specially in endemic areas of TB, otherwise it would lead to misdiagnosis with dangerous consequences.

In this context, Flores-Suárez et al. [16] studying 45 TB patients in Mexico found a prevalence of ANCA of $44 \%$ by IIF, mainly of the cytoplasmic pattern. These authors also found an astonishing positivity of $40 \%$ in their cohort, for anti-PR 3 and anti-MPO antibodies by ELISA. Pradhan et al. [11] demonstrated a positivity of ANCA by IIF, anti-MPO and anti-PR3 by ELISA in 30, 47.6 and $28.6 \%$, respectively, in their cohort of patients with TB. Ghosh et al. [13] gathered the findings obtained by Pradhan et al. with those retrieved from two other studies summing up a total of 318 TB patients. ANCA were positive in $30 \%$ of the entire studied population. Recently, Sherkat et al. evaluated the prevalence of ANCA by IIF, anti-MPO and anti-PR3 antibodies in 32 subjects with active TB comparing with 32 healthy controls. They found P-ANCA and C-ANCA in 25 and $3.1 \%$ of the cases, respectively, mainly due to the presence of anti-MPO antibodies [12]. Another recent study showed 
very intriguing results with most $\mathrm{TB}$ patients having high baseline anti-PR3, anti-MPO and anti-lactoferrin levels. After treatment, most anti-lactoferrin and anti-MPO levels decreased, while anti-PR3 increased. Some patients had de novo anti-PR3 and anti-MPO positivity after treatment [17].

On the other hand, other studies, apart from the present one, have demonstrated an absence or low frequency of positivity of these antibodies in TB. Teixeira et al. [18] found only $10 \%$ of ANCA positivity by IIF in 67 TB patients, and only one had antibodies to PR3. Esquivel-Valerio et al. searched ANCA by IIF, anti-PR3 and anti-MPO in 68 TB patients before treatment and in 52 of them, 60-90 days after initiation of anti-TB therapy. In the pre-treatment samples, the positivity of ANCA by IIF found was 3/68 (4.4\%), being one C-ANCA and two P-ANCA. None of the samples had anti-PR3 or anti-MPO antibodies. Whereas after initiation of treatment, ANCA were identified in 15/52 (28.8\%) being twelve P-ANCA and three C-ANCA. In 11 of these 15 samples $(73.3 \%)$, there was a specificity of the antibodies to bactericidal/permeability-increasing protein. Anti-PR3 and antiMPO antibodies were negative in all tested samples [19].

The findings of the present study, demonstrating the negativity of ANCA by IIF, anti-PR3 and anti-MPO antibodies by ELISA in TB, confirm the high specificity of these tests for the identification of vasculitic syndromes. This observation has been corroborated by the results of a previous study from our institution demonstrating very low prevalence of these antibodies in Hansen's disease [20].

The discrepancy in the prevalence of ANCA in TB observed in different studies may be attributed to methodological factors such as inclusion of patients with bacteriologically unproved TB, or at a later stage of treatment and use of diagnostic kits from unreliable sources. As addressed in a recently published review, lack of standardization of the current assays is one of the problems with the use of ANCA testing in the daily clinical practice [21]. Moreover, the possibility of associated chronic bacterial infections and/or difference in the genetic background of the studied populations cannot be excluded.

Acknowledgments M.B.S., A.A. and M.G.R. receive a scholarship from the "Conselho Nacional de Desenvolvimento Científico e Tecnológico" (CNPq). We are indebted to the pneumologists of Hospital Otávio Mangabeira where the patients came from, for their assistance.

Conflict of interest The authors have no conflict of interest that is directly relevant to the content of this manuscript.

\section{References}

1. Hagen EC, Daha MR, Hermans J, Andrassy K, Csernok E, Gaskin G, Lesavre P, Ludemann J, Rasmussen N, Sinico RA, Wiik A, van der Woude FJ (1998) Diagnostic value of standardized assays for anti-neutrophil cytoplasmic antibodies in idiopathic systemic vasculitis. EC/BCR project for ANCA assay standardization. Kidney Int 53(3):743-753. doi:10.1046/j.1523-1755.1998.00807.x

2. Rother E, Schochat T, Peter HH (1996) Antineutrophil cytoplasmic antibodies (ANCA) in rheumatoid arthritis: a prospective study. Rheumatol Int 15(6):231-237

3. Pradhan VD, Badakere SS, Bichile LS, Almeida AF (2004) Antineutrophil cytoplasmic antibodies (ANCA) in systemic lupus erythematosus: prevalence, clinical associations and correlation with other autoantibodies. J Assoc Physicians India 52:533-537

4. Romas E, Paspaliaris B, d'Apice AJ, Elliott PR (1992) Autoantibodies to neutrophil cytoplasmic (ANCA) and endothelial cell surface antigens (AECA) in chronic inflammatory bowel disease. Aust N Z J Med 22(6):652-659

5. Pradhan V, Badakere SS, Shankar Kumar U (2004) Increased incidence of cytoplasmic ANCA (cANCA) and other autoantibodies in leprosy patients from western India. Lepr Rev 75(1):50-56

6. Pradhan V, Badakere SS, Shankarkumar U, Iyer YS, Ghosh K, Karnad D (2002) Anti-neutrophil cytoplasmic antibodies (ANCA) in malaria. Indian J Malariol 39(3-4):51-59

7. Medina F, Camargo A, Moreno J, Zonana-Nacach A, AcevesAvila J, Fraga A (1998) Anti-neutrophil cytoplasmic autoantibodies in leprosy. Br J Rheumatol 37(3):270-273

8. Soto A, Jorgensen C, Oksman F, Noel LH, Sany J (1994) Endocarditis associated with ANCA. Clin Exp Rheumatol 12(2):203-204

9. Habegger de Sorrentino A, Motta P, Iliovich E, Sorrentino AP (1997) Anti-neutrophil cytoplasmic antibodies (ANCA) in patients with symptomatic and asymptomatic HIV infection. Medicina 57(3):294-298

10. Bonaci-Nikolic B, Andrejevic S, Pavlovic M, Dimcic Z, Ivanovic B, Nikolic M (2010) Prolonged infections associated with antineutrophil cytoplasmic antibodies specific to proteinase 3 and myeloperoxidase: diagnostic and therapeutic challenge. Clin Rheumatol 29(8):893-904. doi:10.1007/s10067-010-1424-4

11. Pradhan VD, Badakere SS, Ghosh K, Pawar AR (2004) Spectrum of anti-neutrophil cytoplasmic antibodies in patients with pulmonary tuberculosis overlaps with that of Wegener's granulomatosis. Indian J Med Sci 58(7):283-288

12. Sherkat R, Mostafavizadeh K, Zeydabadi L, Shoaei P, Rostami S (2011) Antineutrophil cytoplasmic antibodies in patients with pulmonary tuberculosis. Iran J Immunol IJI 8(1):52-57. doi:IJIv8i1A7

13. Ghosh K, Pradhan V, Ghosh K (2008) Background noise of infection for using ANCA as a diagnostic tool for vasculitis in tropical and developing countries. Parasitol Res 102(5):1093-1095. doi:10.1007/s00436-008-0929-0

14. Davies DJ, Moran JE, Niall JF, Ryan GB (1982) Segmental necrotising glomerulonephritis with antineutrophil antibody: possible arbovirus aetiology? Br Med J (Clin Res Ed) 285(6342):606

15. van der Woude FJ, Rasmussen N, Lobatto S, Wiik A, Permin H, van Es LA, van der Giessen M, van der Hem GK, The TH (1985) Autoantibodies against neutrophils and monocytes: tool for diagnosis and marker of disease activity in Wegener's granulomatosis. Lancet 1(8426):425-429

16. Flores-Suarez LF, Cabiedes J, Villa AR, van der Woude FJ, Alcocer-Varela J (2003) Prevalence of antineutrophil cytoplasmic autoantibodies in patients with tuberculosis. Rheumatology (Oxford) 42(2):223-229

17. Elkayam O, Bendayan D, Segal R, Shapira Y, Gilburd B, Reuter S, Agmon-Levin N, Shoenfeld Y (2013) The effect of anti-tuberculosis treatment on levels of anti-phospholipid and anti-neutrophil cytoplasmatic antibodies in patients with active tuberculosis. Rheumatol Int 33(4):949-953. doi:10.1007/s00296-012-2487-0

18. Teixeira L, Mahr A, Jaureguy F, Noel LH, Nunes H, Lefort A, Barry S, Deny P, Guillevin L (2005) Low seroprevalence and 
poor specificity of antineutrophil cytoplasmic antibodies in tuberculosis. Rheumatology (Oxford) 44(2):247-250. doi:10.1093/ rheumatology/keh467

19. Esquivel-Valerio JA, Flores-Suarez LF, Rodriguez-Amado J, Garza-Elizondo MA, Rendon A, Salinas-Carmona MC (2010) Antineutrophil cytoplasm autoantibodies in patients with tuberculosis are directed against bactericidal/permeability increasing protein and are detected after treatment initiation. Clin Exp Rheumatol 28(1 Suppl 57):35-39
20. Edington FL, Bacellar MO, Machado PR, Barbosa L, Reis E, Reis M, Santiago MB (2007) Anti-neutrophil cytoplasmic antibodies in leprosy. Clin Rheumatol 26(2):208-210. doi:10.1007/ s10067-006-0281-7

21. Radice A, Bianchi L, Sinico RA (2013) Anti-neutrophil cytoplasmic autoantibodies: methodological aspects and clinical significance in systemic vasculitis. Autoimmun Rev 12(4):487-495. doi:10.1016/j.autrev.2012.08.008 